\title{
Radiological Analysis of the Triangular Working Zone during Transforaminal Endoscopic Lumbar Discectomy
}

\author{
Erdinc Civelek ${ }^{1}$, Ilker Solmaz ${ }^{1}$, Tufan Cansever ${ }^{2}$, Bulent Onal ${ }^{1}$, \\ Serdar Kabatas ${ }^{2}$, Nail Bolukbasi ${ }^{3}$, Sait Şirin ${ }^{1}$, Serdar Kahraman ${ }^{1}$ \\ ${ }^{1}$ Department of Neurosurgery, Gulhane Military Medical Academy, Ankara, Turkey \\ ${ }^{2}$ Department of Neurosurgery, Baskent University Istanbul Hospital, Istanbul, Turkey \\ ${ }^{3}$ Department of Radiology, Gulhane Military Medical Academy, Ankara, Turkey
}

Study Design: Clinical study.

Purpose: The dimensions of the working zone for endoscopic lumbar discectomy should be evaluated by preoperative magnetic resonance images. The aim of this study was to analyze the angle of the roots, root area, and foraminal area.

Overview of Literature: Few studies have reported on the triangular working zone during transforaminal endoscopic lumbar discectomy. Many risk factors and restrictions for this procedure have been proposed.

Methods: Images of 39 patients were analyzed bilaterally at the levels of L3 L4 and L4 L5. Bilateral axial and coronal angles of the roots, root area, and foraminal area were calculated.

Results: No significant difference was observed between the axial angle of the left and right L3 root. A significant difference was found between the axial angle of right and left L4 roots. A significant difference was observed when the coronal angle of the right and left L3 roots were compared,but no significant difference was found when the coronal angle of the right and left L4 roots were compared. No significant difference was observed when the foraminal area of the right and left L3 and L4 roots were compared, but a significant difference was observed when the root area of right and left L3 and L4 roots were compared.

Conclusions: We suggest that these radiological measurements should be obtained for safety reasons before endoscopic discectomy surgery.

Key Words: Triangular working zone, Endoscopy, Lumbar

\section{Introduction}

Lower back pain is the most common cause of chronic incapacity in adults under 45-years-of-age and is one of the most frequent causes of early retirement in industrialized countries [1,2]. One of the most frequent causes of lower back pain is lumbar disc herniation.

The extensive soft tissue and bone manipulation involved in a classical discectomy motivated a number of indepen- dent authors to propose an alternative microdiscectomy approach for treating lumbar radiculopathy [3,4]. In 1993, Mayer and Brock [5], and in 1997, Foley and Smith [6] described endoscopic discectomy techniques. These authors proposed that the use of an endoscope shortened operating time and hospital stay and improved early recovery.

During these advances in lumbar disc herniation surgical techniques, the triangular working zone has been identified as a safe zone in the posterolateral corner of the interver-

Received Jun 8, 2011 Revised Aug 11, 2011 Accepted Aug 25, 2011

Corresponding author: Erdinc Civelek, MD

Department of Neurosurgery, Gulhane Military Medical Academy, Etlik, Ankara, Turkey

Tel: +90 532326 2494, Fax: +90 312304 5316, E-mail: civsurgeon@yahoo.com 
tebral disc, which allows safe passage of instruments with minimal risk to the exiting nerves. The triangular working zone may be topographically described as the area within a right-angle triangle in which the inferior border is formed by the rim of the vertebral plate inferior to the target disc, the posterior border is formed by the lateral edge of the superior articular process of the next inferior vertebra, and the hypotenuse is provided by the medial border of the associated spinal nerve as it exits the foramen [7,8]. Mirkovic et al. [9] and Min et al. [10] defined the anatomical borders
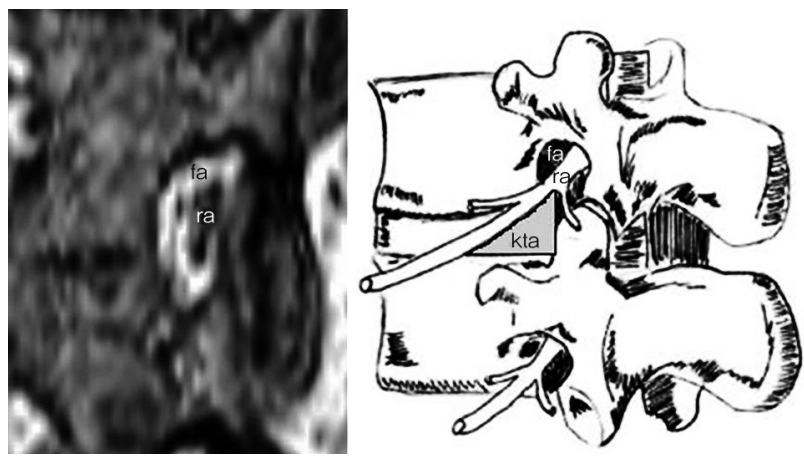

Fig. 1. Calculation of foraminal and radicular area. fa: Foraminal area, ra: Radicular area, kta: Kambin triangle.

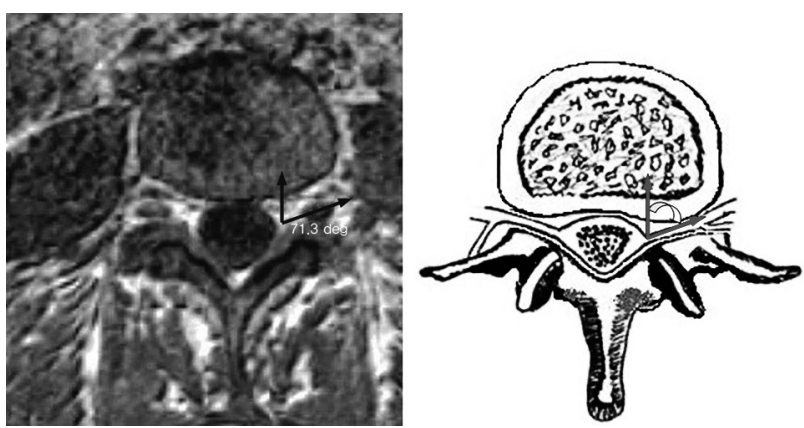

Fig. 2. An example of calculating the root axial angle. $r$ : Root.
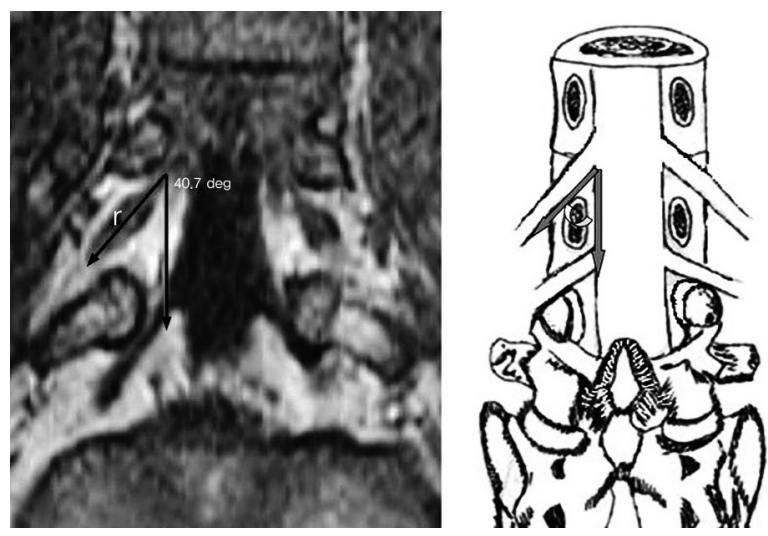

Fig. 3. An example of calculating the root coronal angle. $r$ : Root. and dimensions of the working zone. Their morphometric study focused on the coronal and sagittal plane dimensions. Great care must be taken at this triangle during surgical procedures, yet studies concerning the dimensions of this working zone are rare. The dimensions of the working zone must be evaluated radiologically by magnetic resonance images (MRI) prior to endoscopic lumbar discectomy. The purpose of this study was to analyze the working zone at the lateral exit zone of the intervertebral foramen and to provide data associated with the working zone to help endoscopists by analyzing a series of MRIs.

\section{Materials and Methods}

All measurements provided in this study were collected independently by two experienced radiologists. They measured the same parameters twice, and the mean values were calculated. Images were acquired using a 1.5 T MR scanner (New Intera Nova, Philips Medical Systems, Best, The Netherlands) with a standard spine coil and version 9 release software. The system was equipped with magnetic field gradients capable of a maximum strength of $33 \mathrm{mT} / \mathrm{m}$ and a slew rate of $150 \mathrm{~T} / \mathrm{m} / \mathrm{s}$. Axial TFE T1 weighted (shortest TE, $25 \mathrm{~ms}$ TR) sequences were acquired using a $1 \mathrm{~mm}$ slice thickness, $150 \mathrm{~mm}$ rectangular field of view, NSA of 2, and 75 slices including the L3-L5 area, in a total acquisition time of $6,06 \mathrm{~min}$. The imaged voxel size was $0.59 / 0.77 / 2$ $\mathrm{mm}$ and the reconstructed voxel size was $0.59 / 0.59 / 1 \mathrm{~mm}$. Image data were analyzed on an expanded workstation (Philips, Easy Vision with release 5.2.2.1) using the Volume View package. MRI was used for image analysis at the level of L3-L4 and L4-L5 disks. Thirty-nine MRIs from 39 randomized patients with a complaint of low back pain (ages, 30-50 years with no lumbar operation history) were obtained and analyzed bilaterally at the levels of L3-L4 and L4-L5 discs. The foramen area was calculated from previous bilateral slices in a sagittal plane that passed through the exit of the foramen, with virtual dots on the inner border of the foramen. In the same plane, the root area was calculated bilaterally using virtual dots of the root outer border. Then, the root was seen completely in the foramen in the axial and coronal plane, and the axial angle was measured bilaterally between virtual lines that pass-through the spinous processes and the root center in parallel. The coronal angle was measured bilaterally between virtual lines that pass through the spinal cord and root center in parallel (Figs. 1-3).

Statistical analyses were performed using SPSS Windows 
Table 1. Descriptive values of statistics

\begin{tabular}{|c|c|c|c|}
\hline & Min. & Max. & Mean \pm SD \\
\hline \multicolumn{4}{|c|}{ Axial angle $\left(^{\circ}\right)$} \\
\hline RL3 & 39.00 & 87.00 & $64.41 \pm 11.58$ \\
\hline LL3 & 48.00 & 85.00 & $66.56 \pm 8.83$ \\
\hline RL4 & 44.00 & 88.00 & $67.74 \pm 11.87$ \\
\hline LL4 & 47.00 & 92.00 & $66.87 \pm 10.23$ \\
\hline \multicolumn{4}{|c|}{ Coronal angle $\left(^{\circ}\right)$} \\
\hline RL3 & 23.00 & 56.00 & $37.41 \pm 7.12$ \\
\hline LL3 & 24.00 & 60.00 & $35.46 \pm 7.98$ \\
\hline RL4 & 20.00 & 61.00 & $39.20 \pm 8.63$ \\
\hline LL4 & 25.00 & 89.00 & $42.51 \pm 13.14$ \\
\hline \multicolumn{4}{|c|}{ Foraminal area $\left(\mathrm{mm}^{2}\right)$} \\
\hline RL3 & 91.00 & 250.00 & $140.07 \pm 38.19$ \\
\hline LL3 & 69.00 & 250.00 & $137.94 \pm 35.59$ \\
\hline RL4 & 71.00 & 194.00 & $121.00 \pm 32.88$ \\
\hline LL4 & 68.00 & 210.00 & $121.89 \pm 32.42$ \\
\hline \multicolumn{4}{|c|}{ Root area $\left(\mathrm{mm}^{2}\right)$} \\
\hline RL3 & 10.00 & 52.00 & $23.14 \pm 8.97$ \\
\hline LL3 & 13.00 & 49.00 & $24.61 \pm 8.22$ \\
\hline RL4 & 10.00 & 73.00 & $24.46 \pm 10.55$ \\
\hline LL4 & 12.00 & 63.00 & $24.71 \pm 9.65$ \\
\hline
\end{tabular}

Min.: Minimum, Max.: Maximum, SD: Standard deviation, R: Right, L: Left.

ver. 11.5 (SPSS Inc., Chicago, IL, USA). Descriptive statistics (mean, standard deviation, maximum, and minimum) were used. The two-tailed Pearson's correlation test was used to test for correlations between the groups. A one-way analysis of variance was used to compare differences within and between the groups. Probability values of $p<0.05$ and $p<0.01$ were considered statistically significant depending on the variable. Inter-rater and intra-rater reliability between the two radiologists and their two computer-aided measures were analyzed using Kappa statistics for categorical variables.

\section{Results}

Evaluation of the axial angle of the right L3 root in 39 patients was $64.41 \pm 11.58$ and $66.56 \pm 8.83$ for the left L3 root. No significant difference was observed between the axial angles of the left and right roots $(p=0.116)$. But, when the axial angle of the right $(67.74 \pm 11.87)$ and left $(66.87 \pm$ 10.23) L4 roots were compared, a significant $(p=0.006)$ dif- ference was observed (Fig. 4A).

A significant difference was observed when the coronal angle of the right $(37.41 \pm 7.12)$ and left $(35.46 \pm 7.98) \mathrm{L} 3$ roots were compared ( $p=0.001)$, whereas no significant difference was found when the coronal angle of the right $(39.20 \pm 8.63)$ and left $(42.51 \pm 13.14)$ L4 roots were compared $(p=0.418)$ (Fig. 4B).

No significant difference was observed when the foraminal area of the right $(140.07 \pm 38.19)$ and left $(137.94 \pm$ $35.59)$ L3 roots were compared ( $p=0.109)$. Additionally, no significant difference was observed when the foraminal area of the right $(121.00 \pm 32.88)$ and left $(121.89 \pm 32.42)$ L4 roots were compared ( $p=0.700)$ (Fig. 4C).

A significant difference was observed when the root area of the right $(23.14 \pm 8.97)$ and left $(24.61 \pm 8.22) \mathrm{L} 3$ roots were compared $(p<0.0001)$. A significant difference was also observed when the root area of the right $(24.46 \pm$ $10.55)$ and left $(24.71 \pm 9.65) \mathrm{L} 4$ roots were compared $(p=$ 0.009) (Fig. 4D).

No correlation was found between the coronal angle 
Table 2. Kappa coefficients for inter (between two radiologists)-and intra (between two measures of the same radiologist)rater reliability in determining of axial angle (AA), coronal angle (CA), foraminal area (FA) and root area (RA)

\begin{tabular}{lccccc}
\hline \hline & \multicolumn{2}{c}{ Inter-rater reliability } & & \multicolumn{2}{c}{ Intra-rater reliability } \\
\cline { 2 - 3 } \cline { 5 - 5 } & \% Agreement & Kappa & & \% Agreement & Kappa \\
\hline RL3 AA & 98.2 & 0.95 & 99.1 & 0.98 \\
LL3 AA & 97.5 & 0.94 & & 97.4 & 0.96 \\
RL4 AA & 98.7 & 0.96 & & 99.3 & 0.98 \\
LL4 AA & 95.4 & 0.93 & & 96.8 & 0.94 \\
RL3 CA & 96.6 & 0.94 & & 98.4 & 0.96 \\
LL3 CA & 94.1 & 0.90 & & 97.9 & 0.96 \\
RL4 CA & 96.3 & 0.93 & & 98.8 & 0.97 \\
LL4 CA & 95.7 & 0.92 & & 99.2 & 0.98 \\
RL3 FA & 91.1 & 0.88 & & 97.6 & 0.96 \\
LL3 FA & 92.6 & 0.89 & & 95.4 & 0.93 \\
RL4 FA & 95.3 & 0.91 & & 96.6 & 0.95 \\
LL4 FA & 94.2 & 0.90 & & 96.4 & 0.94 \\
RL3 RA & 93.8 & 0.89 & & 95.3 & 0.92 \\
LL3 RA & 92.6 & 0.88 & & 96.1 & 0.93 \\
RL4 RA & 94.7 & 0.90 & & 98.8 & 0.97 \\
LL4 RA & 94.1 & 0.89 & & 95.6 & 0.94 \\
\hline
\end{tabular}

R: Right, L: Left.
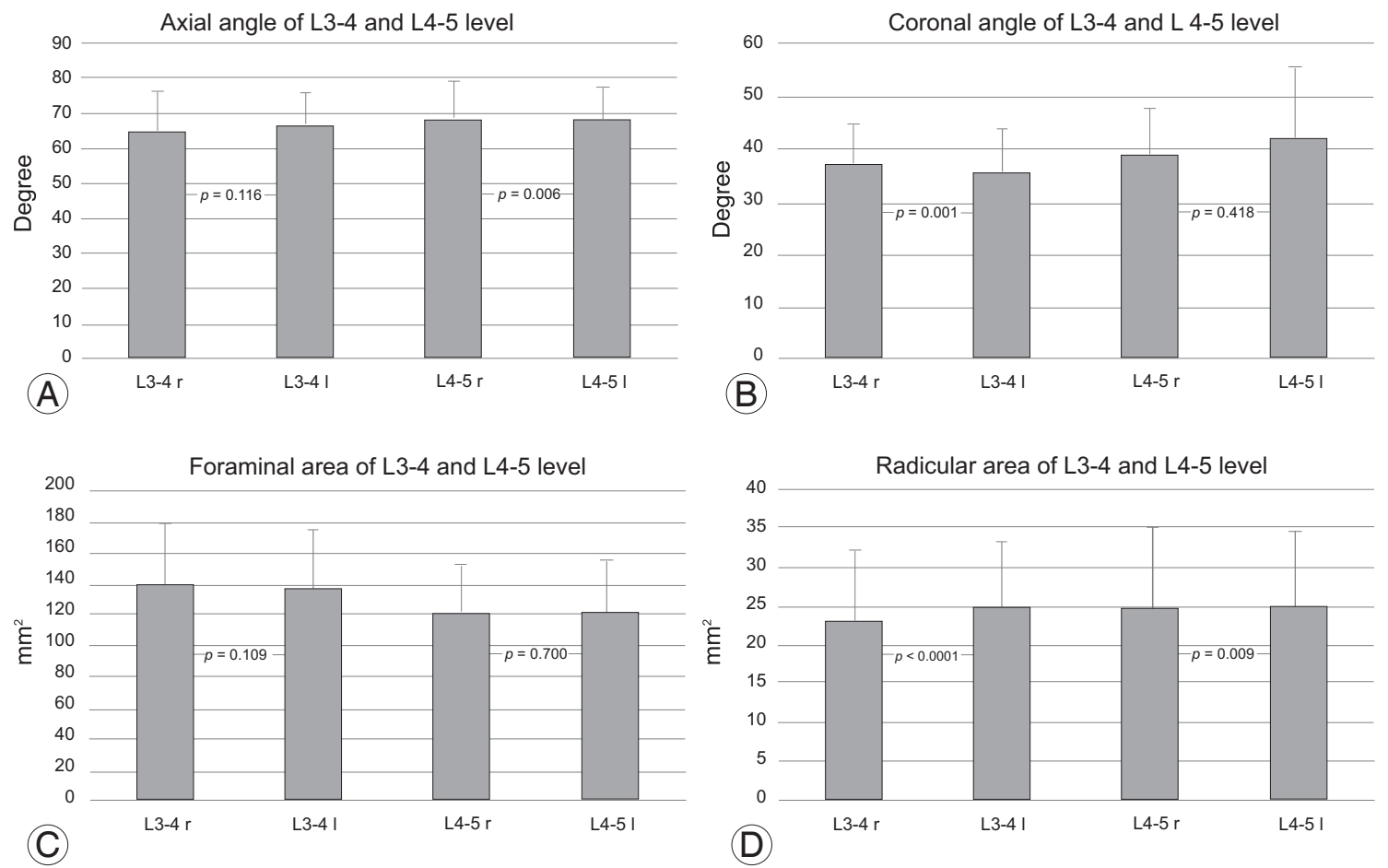

Fig. 4. Schematic presentation of the correlations between axial and coronal angles of the roots and foraminal and radicular areas. r: Right, 1: Left. 
of the left L3 root and the right L3 $(p=0.178)$ and L4 ( $p=0.193)$ roots. No correlations were found when other angles and areas were tested. Descriptive values of the statistics are summarized in Table 1.

There was high intra-rater reliability (between two measures of the same radiologist) among all kappa coefficients ranging from 0.92 to 0.98 . There was high agreement between the two radiologists (inter-rater reliability) computer aided measures with kappa values ranging from 0.88 to 0.96 (Table 2).

\section{Discussion}

The aim of surgical treatment for a lumbar disc herniation is sufficient decompression and minimizing operationinduced trauma. A faster recovery is needed in modern society, allowing patients to resume their normal activities sooner. Technical developments in the past decades have made treatment of herniated discs safer and less invasive. By using microsurgical or microendoscopic techniques through small incisions, nerve root decompression is performed with minimal complication risk and preserves normal anatomy.

Endoscopic lumbar discectomy (ED) is growing in popularity for treating disc herniation, and the range of indications has expanded with advances in instruments including endoscopes. ED has several theoretical advantages over conventional open surgery. Most current percutaneous endoscopic discectomy techniques are based on the Kambin's transforaminal approach and offer favorable outcomes for soft disc herniation. The most important point of successful endoscopic treatment is an accurate approach for the proper indication.

Transforaminal endoscopic discectomy has many advantages over conventional open discectomy such as shorter hospital stay and less postoperative pain medication [11-14]. Transforaminal endoscopic discectomy is not indicated for patients with severe spinal stenosis, cauda equina syndrome, or disc herniations with highly migrated sequestrated fragments. But, operating on a disc herniation between the fifth lumbar and first sacral segment using an endoscopic technique in individuals with a high iliac crest may be difficult. Sometimes endoscopic discectomy may not be performed because of the anatomical relationship between the foramen and the root and root irritation [15].

The transforaminal endoscopic discectomy approach begins at the skin entry point at the posterolateral aspect of the lower back ( 8 to $14 \mathrm{~cm}$ from the midline) and proceeds through the critical triangular window of the intervertebral foramen, which is bordered superolaterally by the exiting nerve root, posteriorly by the superior articular process of the facet joint, and caudally by the inferior pedicle. During this procedure, the patient should be kept continuously awake under local anesthesia and instructed to report any pain, numbness, or electrical shock sensations to prevent nerve injuries.

The available angle to reach around the superior boundary of the foramen is much larger than that around the inferior boundary of the foramen. Therefore, this anatomical characteristic allows endoscopic instruments a sufficient angle to pass through the foramen into the upper portion of the spinal canal and easily remove cephalad-migrated disk material. In contrast, the inferior boundary of the foramen limits reaching the spinal canal below the disk level in cases of a caudal-migrated disk herniation. Over-manipulation of spinal canal content and compression of the exiting root and ganglia by a horizontally positioned cannula may cause neural injury, as well as postoperative pseudocausalgic pain in the index extremity. The surgical approach has evolved from the traditional posterolateral approach into a transforaminal approach. Thus, confirmation of the dimensions of the working zone appropriate for the transforaminal approach is required [10].

Min et al. [10] concluded that the mean diameter of the working zone base was $11.6 \pm 4.6 \mathrm{~mm}$ and this value increases going down the level of the spine. In the same study performed on cadaveric spines, the L2-L3, L3-L4, and L4L5 intervertebral foramens were similar in multiple comparison tests. Similarly, in the current study, foraminal area of the right L3-L4 foramen was $140.07 \pm 38.19 \mathrm{~mm}^{2}$ and was $137.94 \pm 35.59 \mathrm{~mm}^{2}$ on the left. The foraminal area of the right L4-L5 foramen was $121.00 \pm 32.88 \mathrm{~mm}^{2}$ and was $121.89 \pm 32.42 \mathrm{~mm}^{2}$ on the left. In our study, comparisons between both sides were performed, and no significant difference was observed between these parameters. So, there would be no technical difference when performing ED at these levels.

The proportion of root area to foraminal area can be used to predict the efficacy of the endoscopic discectomy procedure for each particular case. This can be easily performed by assessing a preoperative MRI.

The working cannula should be inserted into the foramen as close as possible to the facet joint and not directly target the disc. Ahn et al. [16] and Min et al. [10] have confirmed 
this method.

The superior and inferior borders of the working zone are formed by a line from the posterior margin of the spinal nerve root to the superior articular process parallel to the inferior and superior endplate. The anterior border is formed by the exiting root and is the oblique side. In a study performed on cadaveric spines by Min et al. [10], the average angle between the anterior border and the base was $79.1^{\circ} \pm$ $7.6^{\circ}$. This value decreased going down the spine level. In that study, they analyzed the working zone dimensions at the lateral exit zone of the intervertebral foramen, but the working zone is actually a three-dimensional structure. That study was limited, because they analyzed the working zone in only two dimensions. Additionally, deformation of the spinal nerve roots in cadaveric spines may have had a negative effect. In our study, the root angles were calculated in both axial and coronal planes. Although a significant difference was observed in the coronal L3 root angles and axial L4 root angles of the right and left sides, no difference was found in the root angles at these two disc levels. In contrast to cadaveric studies $[9,10]$, the values we found were small. But, our study showed no significant differences between individuals that may create technical difficulties for a transforaminal endoscopic lumbar discectomy.

Transforaminal endoscopic lumbar discectomy may be used for upper lumbar disc herniations [17]. A radiological analysis of the upper lumbar region before the operation may be useful to perform a safer procedure.

One of the limitations of this study was the absence of intraoperative data. A correlation between preoperative MRI measurements and intraoperative data would be more valuable. Although the current study was a radiological study, the data given here may help surgeons plan the procedure by evaluating endoscope diameter and foraminal and root area.

\section{Conclusions}

The working zone dimensions have clinical significance in the practice of endoscopic discectomy. Our data suggest that radiological measurements including the spinal root axial and coronal angle and foraminal areas should be obtained before endoscopic discectomy surgery to provide for a safe procedure. This radiological study showed some anatomical differences to improve the approach and decrease the incidence of complications. Our study showed a significant difference between the levels and sides of the root axial and coronal angles, root areas, and foraminal areas.

All studies in the literature regarding this subject were conducted using formalin-fixed cadavers or lumbar spines that were chemically processed $[9,10]$. Formalin-fixation techniques render tissue structures soft. In contrast to other studies, the current study was performed using MRIs of living individuals; therefore we believe that our data were more accurate and helpful for the surgical approach.

\section{REFERENCES}

1. Heliövaara M, Impivaara O, Sievers K, et al. Lumbar disc syndrome in Finland. J Epidemiol Community Health 1987;41:251-8.

2. Williams KD, Park AL. Lower back pain and disorders of intervertebral discs. In: Canale ST, editor. Campbell's operative orthopaedics. St. Louis: Mosby; 2003. p.1955-2028.

3. Williams RW. Microlumbar discectomy: a conservative surgical approach to the virgin herniated lumbar disc. Spine (Phila Pa 1976) 1978;3:175-82.

4. Yaşargil MG. Microsurgical operation of herniated lumbar disc. Adv Neurosurg 1977;4:81-2.

5. Mayer HM, Brock M. Percutaneous endoscopic discectomy: surgical technique and preliminary results compared to microsurgical discectomy. J Neurosurg 1993;78:216-25.

6. Foley KT, Smith MM. Microendoscopic discectomy. Tech Neurosurg 1997;4:301-7.

7. Ditsworth DA. Endoscopic transforaminal lumbar discectomy and reconfiguration: a postero-lateral approach into the spinal canal. Surg Neurol 1998;49:588-97.

8. Parke WW. Anatomy of the spinal nerve and its surrounding structures. Semin Orthop 1991;6:65-71.

9. Mirkovic SR, Schwartz DG, Glazier KD. Anatomic considerations in lumbar posterolateral percutaneous procedures. Spine (Phila Pa 1976) 1995;20:1965-71.

10. Min JH, Kang SH, Lee JB, Cho TH, Suh JK, Rhyu IJ. Morphometric analysis of the working zone for endoscopic lumbar discectomy. J Spinal Disord Tech 2005;18:132-5.

11. Hermantin FU, Peters T, Quartararo L, Kambin P. A prospective, randomized study comparing the results of open discectomy with those of video-assisted arthroscopic microdiscectomy. J Bone Joint Surg Am 1999;81:958-65.

12. Huang TJ, Hsu RW, Li YY, Cheng CC. Less systemic 
cytokine response in patients following microendoscopic versus open lumbar discectomy. J Orthop Res 2005;23:406-11.

13. Kambin P, Gellman H. Percutaneous lateral discectomy of the lumbar spine. A preliminary report. Clin Orthop 1983;174:127-32.

14. Righesso O, Falavigna A, Avanzi O. Comparison of open discectomy with microendoscopic discectomy in lumbar disc herniations: results of a randomized controlled trial. Neurosurgery 2007;61:545-9.
15. Kafadar A, Kahraman S, Akbörü M. Percutaneous endoscopic transforaminal lumbar discectomy: a critical appraisal. Minim Invasive Neurosurg 2006;49:74-9.

16. Ahn Y, Lee SH, Park WM, Lee HY. Posterolateral percutaneous endoscopic lumbar foraminotomy for L5-S1 foraminal or lateral exit zone stenosis. Technical note. J Neurosurg 2003;99:320-3.

17. Kambin P, Gennarelli T, Hermantin F. Minimally invasive techniques in spinal surgery: current practice. Neurosurg Focus 1998;4:e8. 